\title{
POMzites: A roadmap for inverse design in metal oxide chemistry
}

\author{
Laia Vilà-Nadal $\odot$
}

School of Chemistry, The University of Glasgow, Glasgow, UK

\section{Correspondence}

Laia Vilà-Nadal, School of Chemistry, The University of Glasgow, Glasgow, G12 8QQ UK.

Email: laia.vila-nadal@glasgow.ac.uk

Funding information

Engineering and Physical Sciences Research Council, Grant/Award Number: EP/S030603/1

\begin{abstract}
Computationally exploring the space generated by the self-assembly of known molecular metal oxides and the ability to predict new architectures is a challenging task. As a proof of concept, here, we propose narrowing it down to a new family of all-inorganic porous materials named POMzites. Structures with new topologies, but aiming for pure inorganic systems, will be targeted initially. POMzites are composed of ring-shaped tungsten oxide building blocks connected with transition metal linkers forming zero to three dimensional frameworks. Despite POMzites and zeolites having similar structures, the library of POMzites is an order of magnitude smaller than that of zeolites (14 POMzites vs 213 zeolites). The idea proposed in this perspective article is to accelerate the discovery of new POMzite porous frameworks materials using inverse design approaches.
\end{abstract}

\section{KEYWORDS}

computational chemistry, inverse design, metal oxides, polyoxometalates, porous materials

\section{1 | INTRODUCTION}

Zeolites and metal-organic frameworks (MOFs) are crystalline porous materials that can trap liquid or gas molecules. After intensive research on porous materials, a rational design has been achieved, and to a broader extent, it is possible to specify the size, shape, and uniformity of the pores in both zeolites and MOFs. Early transition metals, W, Mo, or V, assemble into molecular metal oxide clusters, forming a diverse family of compounds named polyoxometalates (POMs). The metal centers in POM clusters are connected through shared oxygen atoms and incorporate additional elements such as Si, P, or Ge. POM clusters are zero-dimensional molecules, but introducing transition metals to POM solutions can result in connectivity between discrete clusters and their extension into three-dimensional (3-D) networks. Since 2017, there is a new family of allinorganic porous materials, POM frameworks named "POMzites", composed of ring-shaped tungsten oxide $\left[\mathrm{P}_{8} \mathrm{~W}_{48} \mathrm{O}_{184}\right]^{40-}(\mathrm{abbreviated} \mathrm{as}$ $\left\{\mathrm{P}_{8} \mathrm{~W}_{48}\right\}$ ) building blocks connected with transition metal linkers (TM-linkers) forming zero- to three-dimensional frameworks; see Figure 1. ${ }^{[1]}$ The choice of $\left\{\mathrm{P}_{8} \mathrm{~W}_{48}\right\}$ as a building block is justified due its robust synthetic method, ${ }^{[2]}$ its high negative charge, remarkable electrochemistry, and its intrinsic porosity. Hence, unlike what happens in zeolites and MOFs, POMzites based on the $\left\{\mathrm{P}_{8} \mathrm{~W}_{48}\right\}$ ring do not need a template to form a porous structure. The coined term "POMzites" reflects their zeolitic nature and their POM-, or molecular metal oxide-, based constituents. Architecturally, they are similar to zeolites (213 recognized structures), but currently, the POMzite library is still small, with only 14 members. POMzite "all-inorganic" frameworks are a promising new material that is able to combine the robustness of inorganic materials with the flexibility of organic frameworks. ${ }^{[3]}$ They are robust, porous, electrochemically active solids with potential as components in molecular electronics and flex circuits. ${ }^{[4]}$ In addition, POMzites are able to uptake small molecules $\left(\mathrm{NH}_{3}, \mathrm{CH}_{3} \mathrm{CHO}\right)$ and structurally change under different humidities with eventual applications as small-molecule volatile organic compounds (VOCs) and humidity sensors. In solution, POMzites can uptake 3-D transition-metal ions,

This is an open access article under the terms of the Creative Commons Attribution License, which permits use, distribution and reproduction in any medium, provided the original work is properly cited.

(C) 2020 The Author. International Journal of Quantum Chemistry published by Wiley Periodicals LLC. 


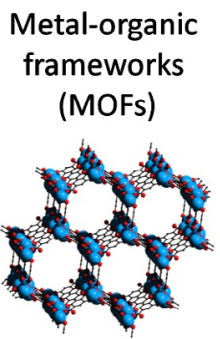

MOF-74

\section{Zeolites}

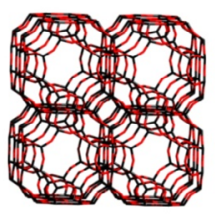

Zeolite A (LTA)
Polyoxometalate-zeolites (POMzites)

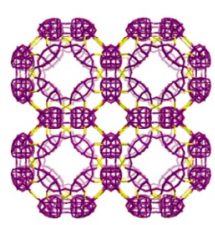

POMzite-3 (Mn)
FIGURE 1 Examples of representative porous materials. Metalorganic frameworks (MOFs), zeolites, and polyoxometalate-zeolites (POMzites)

for example, $\mathrm{Cu}(\mathrm{II})$; small organic molecules; and aromatic organic amines, making them attractive materials for heavy metal uptake and wastewater remediation. ${ }^{[5]}$

Technological revolutions and the discovery of new materials go hand in hand, for instance, the discovery of tungsten light bulb filaments, penicillin, Velcro, Teflon, semiconductors, saccharine, etc. has had a huge impact on society. Some of these discoveries are the result of goaloriented lab work and trial-and-error research, but others are the result of a combination of intuition and serendipity. Easy-to-find materials were found decades ago, and now, we need to dig deep in the materials space to find compounds with the properties that we are looking for. Material science databases and computations are key to finding the next generation of materials; we cannot rely solely on lucky accidents to find new compounds that could transform technology. This perspective presents the recent advances in the development of porous transition metal-oxidebased molecular materials: POMzites. The article is arranged as follows: first an overview on POMzite materials; second the current trends in materials discovery and materials, specifically "inverse design"; third the proposed advances in the area presenting inverse design in POMzites, followed by modeling challenges; and finally, an outlook.

\section{POMzites: POM-BASED FRAMEWORKS}

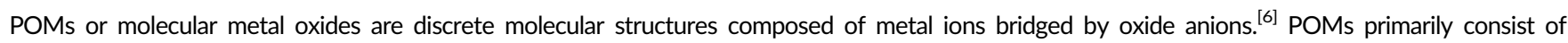
early-transition metal ( $d$-block) elements in their highest oxidation states; a great majority of these structures are anionic and, consequently, salts with charge-balancing cations. They are composed of between 6 and 368 metal ions in a single molecule and are commonly assembled under "one-pot" reaction conditions. POMs, especially tungsto- and molybdophospates and silicates, are widely used in industry for catalysis. ${ }^{[7]}$ Theoretical estimates for new POM materials and their properties will lead to a deeper understanding faster and more efficiently than with test and experimentation alone. First-principles multiscale modeling has allowed a deep understanding of materials by enabling accurate prediction of their electronic properties at a low computational cost, for instance, band gaps that are crucial for memory device applications. ${ }^{[8]}$ Computer simulations have represented a breakthrough in materials science and engineering as simulations offer help in directing experimental efforts. POMs are also called molecular metal oxides due to their molecular composition and their position between monomeric entities and bulk oxides. Despite being well known in the literature for over a 100 years, their self-assembly mechanisms remain semiopaque, and controlling the desired output in a reaction is not always possible. This is because POMs are prepared and isolated from both aqueous and nonaqueous solutions whereby the polymerization of acidified solutions of $\mathrm{Mo}^{\mathrm{VI}}$ or $\mathrm{W}^{\mathrm{VI}}$ yields a chaotic mixture of materials. ${ }^{[9]}$ Preliminary work shows that we can obtain information of the building blocks formed in the synthetic mixtures; hence, we are closer to the design of new metal oxide materials with the targeted properties. As happens with zeolites, the preparation of metal oxide materials can seem like a black art, and predicting their function and synthesis is notoriously difficult. Theoretical methods will be key to studying the derivatization of POM clusters, specifically the $\left\{\mathrm{P}_{8} \mathrm{~W}_{48}\right\}$ cluster, and entangling the mechanism that enables their assembly into a range of frameworks when interacting with TM-linkers. These robust all-inorganic frameworks are synthetized using transition metal-ion linkers (Co, Mn, Ni, Ag), which combine molecular synthetic control without the need for organic components ${ }^{[1,3]}$; see Figure 2 . The resulting POMzite porous materials have high stability and high catalytic, photochemical, and electrochemical activity. Given their novelty, the first publication being in 2017, ${ }^{[1]}$ POMzites are ideal materials to study reaction mechanisms and predict and design new structures. In addition to their porosity and absorption properties, ${ }^{[3]}$ POMzites also provide a flexible framework able to support multicentered redox events, such that charge can be redistributed across the molecule under applied bias; this is a key characteristic in memory device application. ${ }^{[8]}$

\subsection{Current trends in materials design}

Approximately $30 \%$ of the total use of supercomputers at the European level is dedicated to different types of density functional theory (DFT) approximations, and the amount of data generated is truly staggering. ${ }^{[10]}$ Databases of experimental results are highly promising to accelerate catalysis research. Yet, several problems remain, and consensus on issues such as metadata, usability, and healing is key to transform the current excitement into an 


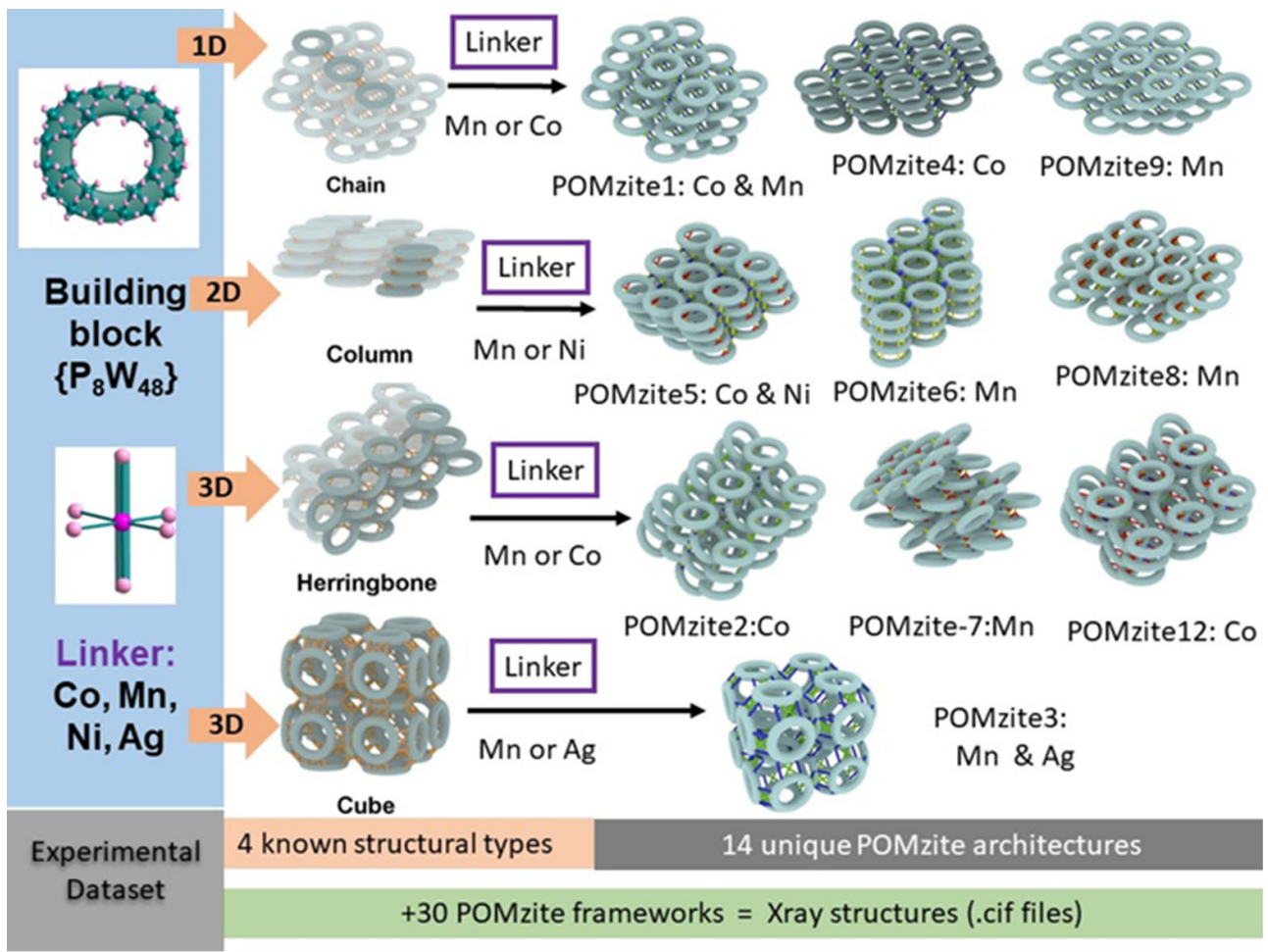

FIGURE 2 The minimal building block library of $\left[\mathrm{P}_{8} \mathrm{~W}_{48} \mathrm{O}_{184}\right]^{40-}$ (abbreviated as $\left.\left\{\mathrm{P}_{8} \mathrm{~W}_{48}\right\}\right)$ nodes and transition metal linkers $(\mathrm{Co}, \mathrm{Mn}, \mathrm{Ni}, \mathrm{Ag})$ that forms a family of 14 POM-all-inorganic framework architectures (namely, "POMzites"). They all share the $\left\{\mathrm{P}_{8} \mathrm{~W}_{48}\right\}$ building-block, with simplified ring representation (green) and have the four structural assembly types: chain (POMzite-4, 9, 10, 14), column (POMzite-5, 6, 8, 11, 13), herringbone (POMzite-2, 7, 12), and cube (POMzite-3). We have selected 10 representative examples of the 14 POMzite architectures described so far. To date, the explored experimental dataset consists of 30 POMzite frameworks, but the accessible chemical space is vast. POMzite structures are reproduced with permission from Boyd et al., ${ }^{[1]}$ American Chemical Society

attractive technology. ${ }^{[10]}$ Material discovery and design efforts ideally involve a close coupling between material prediction, synthesis, and characterization. We need to speed up and lower the cost of the discovery of materials that are able to adapt to the needs of a much more demanding technology. The increased use of computational tools, the generation of materials databases, and advances in experimental methods have substantially accelerated these activities. ${ }^{[11]}$ Among the numerous solutions that have been proposed for this challenge, high-throughput experimental (HTE) methodologies stand out ${ }^{[12]}$ because, for a given material, they are able to quickly establish relationships between composition, structure, and functional properties. Over the past 10 years, ${ }^{[13]}$ HTE methodology has been adopted by material chemists to create large libraries of compounds, allowing a rapid and systematic investigation of new materials. Examples of success of this methodology include the search for: Li-batteries, ${ }^{[14]}$ hydrogen storage, ${ }^{[15]}$ scintillators, ${ }^{[16]}$ electrocatalysts, ${ }^{[17]}$ and two-dimensional (2-D) materials for electronics ${ }^{[18]}$ or to accelerate the discovery of light-absorbing materials. ${ }^{[19]}$ Other solutions in the field of materials by design include: directed simulation, ${ }^{[20]}$ pathway assembly, ${ }^{[21]}$ and inverse design. ${ }^{[22]}$ All these methodologies address similar challenges using large datasets and aim to accelerate the discovery of new materials with targeted properties. In practice, the design of complex materials from the bottom up is very demanding computationally as the number of parameters increases with the complexity of the desired material.

Furthermore, unlike in fullerenes ${ }^{[23]}$ or gold clusters, ${ }^{[24]}$ no topological principles that allow prediction have been found. Common motifs have been identified in many clusters, and POM chemists have been able to manipulate them as a function of the $\mathrm{pH}$, the template, and the linker heteroatom to generate a promising cross-shaped nanomolecular structure. ${ }^{[25]}$ This result represents a game changer in POM chemistry as trapping reactive POM building blocks is the first step of generating libraries with the desired properties that will later assemble into the desired material. Inverse design means targeting the discovery of molecules with a specific application or property. Inverse design in POMzites aims for "top-down" design and "bottom-up" discovery of porous metal oxides to anticipate the formation of extended materials based upon these molecular building blocks. Figure 3 describes a workflow based on expert knowledge of the system, in this case metal oxides, to increase the pace of materials design.

\section{2 | Inverse design in POMzites}

Inverse design could be the gateway to both explore and expand the accessible chemical space generated by the derivatization of POM clusters, which enables their assembly into a range of frameworks using inorganic linkers. ${ }^{[2]}$ Leveraging the decades-long expertise of the broad 


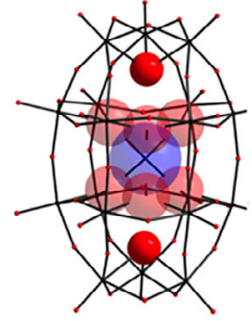

Molecule xyz

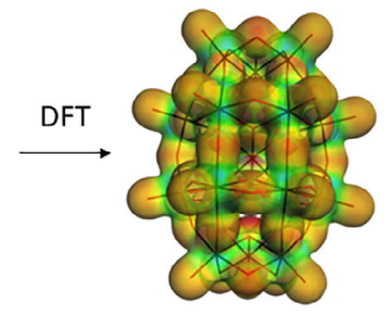

Electronic density $\boldsymbol{\Psi}$
Simulation flow

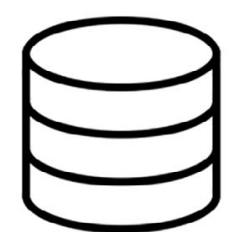

Heterogeneous Database

of known metal oxide materials

Extraction, preprocessing, data reduction

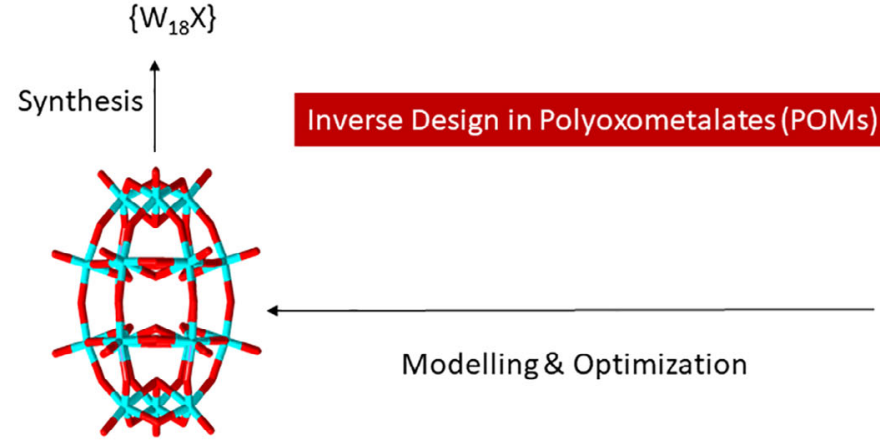

New material

$\left[\mathrm{M}_{18} \mathrm{O}_{56}\left(\mathrm{XO}_{6}\right)\right]^{(4+\mathrm{p})-}$ $\left\{W_{18} X\right\}$
FIGURE 3 Description of the optimized workflow for achieving inverse design in metal oxides. The coordinates of the known molecules are the starting point to generate their ground-state electron density using density functional theory (DFT) methods. Theoretical calculations are used to generate the data points for a structured database combined with an evolutionary design strategy, targeting new metal oxide materials with specific properties. Electronic density image is reproduced with permission from Vilà-Nadal et al. ${ }^{[26]}$ Royal Society of Chemistry

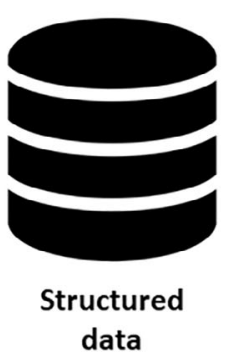

computational material science community ${ }^{[27]}$ and the large amount of data available at the DFT level enables a reliable description of POMzites' electronic structure. ${ }^{[28]}$ To accelerate the discovery of functional nanomolecules and adaptive materials, there is a need to develop theory from aggregated observations using reinforced learning algorithms (see Figure 4). The structural information obtained from electronic structure calculations exploring the chemical space of POMzite materials will map out their reactivity and help to predict new structures and spectra of molecules. ${ }^{[9]}$ The data generated should later be explored and refined using data analysis methods, such as principal component analysis, to establish a new bottom-up approach. This method relies on the analysis of a structural database and the development of a deep neural network to plan for new synthetic routes. Hence, inverse design provides a tool that goes beyond chemical intuition and the serendipitous discovery of new POMzites. This is because it allows us to first predict, and then synthesize, new POMzite materials, establishing a roadmap to discover new POM all-inorganic framework materials, followed by experimental realization.

POMzites are robust all-inorganic frameworks; they are made by combining metal-ion linkers $(\mathrm{Co}, \mathrm{Mn}, \mathrm{Ni}, \mathrm{Ag})$ with $\left\{\mathrm{P}_{8} \mathrm{~W}_{48}\right\}$. This approach combines molecular synthetic control without the need for organic components. ${ }^{[1]}$ Expanding the number of frameworks using inverse design means generating a heterogeneous database of POMzite materials, $1.6 \times 10^{7}$ estimated structures; see Figure 4. For instance, so far, we have just explored four transition metals as linkers, and other linkers could be studied within the $d$-block atoms in the periodic table. With current ab initio calculation packages, we can model POMzites at a molecular level and understand their electronic structure. ${ }^{\text {[27] }}$ Nowadays, it should be computationally cheap to evaluate the stability of a POMzite structure by obtaining its electronic structure through modeling, using ab initio packages with basis sets and functionals for transition metal clusters. ${ }^{[28]}$ The cyclic heteropolyanion $\left[\mathrm{P}_{8} \mathrm{~W}_{48} \mathrm{O}_{184}\right]^{40-}$ (abbreviated as $\left\{\mathrm{P}_{8} \mathrm{~W}_{48}\right\}$ ) is the current building block for the construction of porous framework materials. Preliminary gas-phase calculations confirmed the stability of $\left\{\mathrm{P}_{8} \mathrm{~W}_{48}\right\}$ as an initial structural motif, ${ }^{[29]}$ and derivatives such as the Se equivalent are also stable. A computational setup could provide key data (eg, molecular charge, optimized geometry, HOMO-LUMO gaps, etc.) that can be used as a foundation layer in a neural network. Hence, it is both possible and more sensible to theoretically derivate other cyclic POMs with different heteroatoms (As, $\mathrm{Sb}, \mathrm{S}, \mathrm{Te}$ ) and assess their potential to aggregate into porous structures before starting any attempts to synthesize these new prospective POMzites. Furthermore, POM-based framework materials are rapidly expanding; Schimpf and co-workers have found a new family of oxide frameworks using the Preyssler polyoxoanion $\left[\mathrm{NaP}_{5} \mathrm{~W}_{30} \mathrm{O}_{110}\right]^{14-}$ (abbreviated as $\left\{\mathrm{P}_{5} \mathrm{~W}_{30}\right\}$ ) as a building block. ${ }^{\left[{ }^{30}\right]}$ Framework materials of $\left\{\mathrm{P}_{5} \mathrm{~W}_{30}\right\}$ can be assembled using $\mathrm{Mn}$, Fe, $\mathrm{Co}, \mathrm{Ni}, \mathrm{Cu}$, or $\mathrm{Zn}$ as bridging metal ions. Their result shows the great potential of POMs to form framework materials and validate the cluster assembly as a route to rationally designed and multifunctional complex metal oxide materials. In a further recent study, Schimpf and her team discovered that the competition between $\mathrm{K}^{+}$and $\mathrm{Co}^{2+}$ for binding to $\left\{\mathrm{P}_{5} \mathrm{~W}_{30}\right\}$ dictates the formation of the framework. ${ }^{[31]}$ Their findings provide a deep insight into the factors that govern the formation of metal-bridged POM-based porous networks. 
FIGURE 4 Modeling challenges in generating materials targeting its properties. A proposed workflow to predict new materials by combining machine learning systems with high-level theory using POMzites as a founding case. POMzite structures are reproduced with permission from Boyd et al., ${ }^{[1]}$ American Chemical Society and Zhan et al., ${ }^{[3]}$ Springer Nature

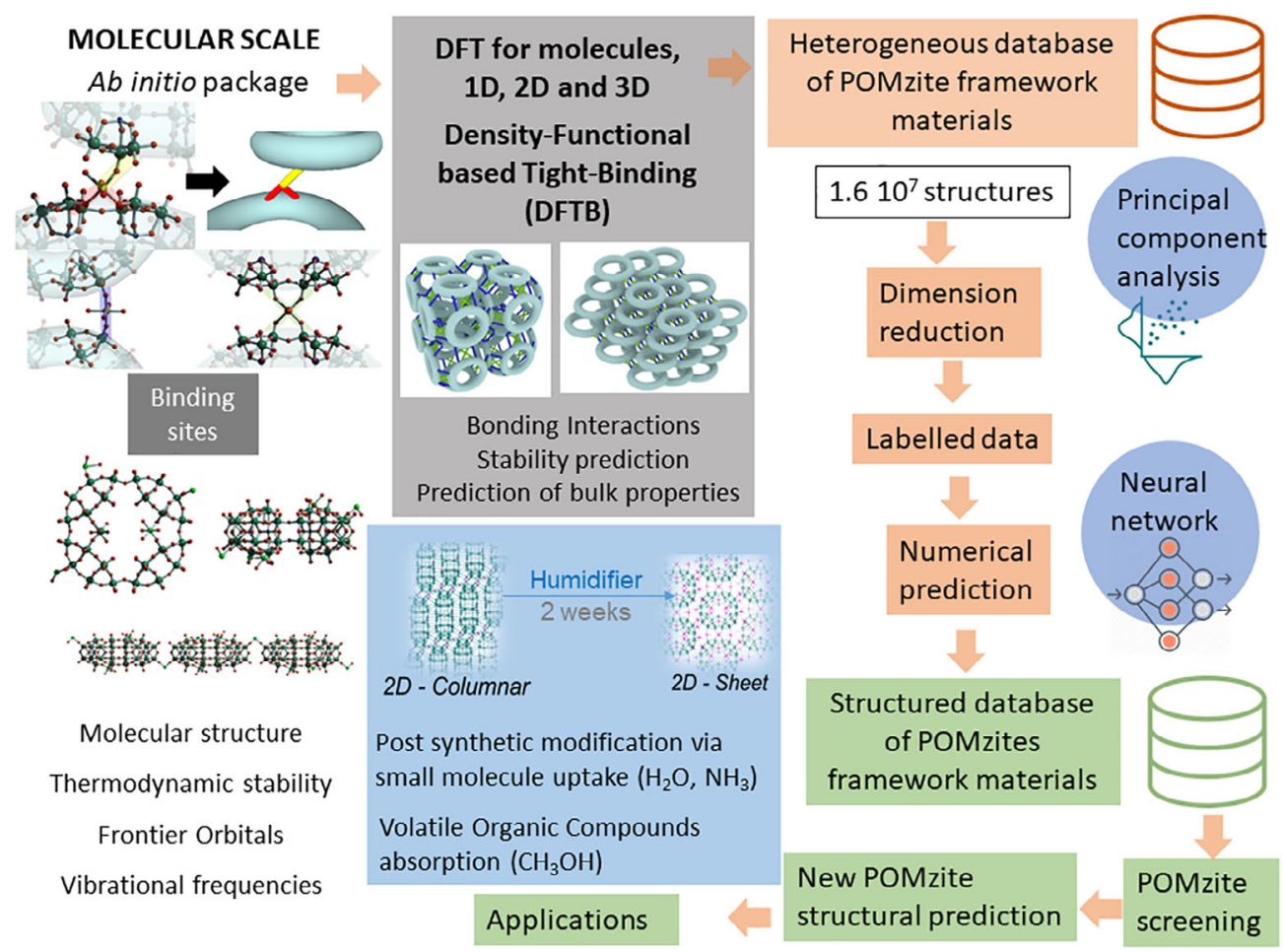

\section{MODELING CHALLENGES}

A challenging aspect of POMzite structures is understanding their binding sites, and this will only be possible by describing their molecular structure. There is also a need to understand the nature of the transition metal coordination to $\left\{\mathrm{P}_{8} \mathrm{~W}_{48}\right\}$ and its extension to POMzite frameworks. Boyd et al. described four types of monodentate coordination modes, where the $\left\{P_{8} W_{48}\right\}$ ring features 64 reactive $\left(W=O_{T}\right)$ reactive sites to which transition metals may possibly bind. ${ }^{[1]}$ Describing $\left\{\mathrm{P}_{8} \mathrm{~W}_{48}\right\}$ reactive sites will help to understand the connectivity and the stability of $\left\{\mathrm{P}_{8} \mathrm{~W}_{48}\right\}$ rings in POMzite structures and predict new motifs from the results, as well as gain the ability to anticipate new favorable structures with different linker modes. Given that some POMzites have just been observed with Co or Ni, for example, POMzite-5, those linkers could be substituted with another transition metal in a model structure. Simple topological methods such as stochastic modeling could be used to make predictions of the different binding sites. This information will be crucial to predict new bulk physical properties from assemblies of these building blocks, for example, electropotential, acidity, optical properties, electronic storage. Once the POMzite chemical system is fully understood, a database of all the molecular architectures could be built with the aim to also predict their physical properties.

In order to apply machine learning models to classify crystalline materials, researchers must choose a descriptor that is able to transform crystal coordinates to real vectors. Claussen et al. acknowledge the difficulty of choosing these descriptors as a nontrivial problem-an accurate descriptor is crucial for the success of a predictive model. ${ }^{[32]}$ Depending on the applications, there are different descriptors available, for instance, Hirn et al. proposed to use Fourier transforms and determinants in the representation model. ${ }^{[33]}$ This type of descriptor is more efficient when aiming to apply machine learning models to molecules rather than using their atomic positions described in Cartesian coordinates. Finding the

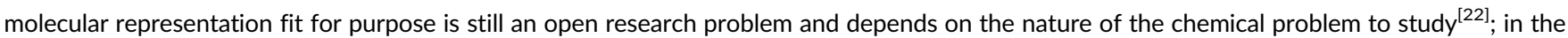
first instance, a reasonable descriptor for POMzites could be its electronic density as it will retain their symmetry.

Beyond classic approaches to molecular-level detailed descriptions, the search for POMzites with targeted properties can also benefit from a modern twist in the ancient art of origami. Overvelde et al. report an algorithm that allows for rational design of origami-inspired materials that can be rearranged to change their properties. ${ }^{[34]}$ Architected materials contain specially engineered structural elements, such as prisms. Here, to design an origami-inspired material, they start identifying a repeating unit composed of polyhedral units. In their work, Overvelde et al. describe an algorithm that not only allows the rational design of reconfigurable prismatic architected materials, it also determines their modes of deformation. ${ }^{[35]}$ This work presents a robust strategy to design 3-D reconfigurable materials that could be extrapolated to POMzites. In fact, it could help to develop an affordable computational model to understand the changes in POMzites crystal structures and their properties. For instance, Zhan et al. described a POMzite $\mathrm{Li}_{9} \mathrm{~K}_{7} \mathrm{~W}_{1} \mathrm{Co}_{10}\left[\mathrm{H}_{2} \mathrm{P}_{8} \mathrm{~W}_{48} \mathrm{O}_{186}\right] 132 \mathrm{H}_{2} \mathrm{O}$ that can undergo at least eight different crystal-to-crystal transformations, without a reduction in crystallinity. ${ }^{[3]}$ Crystals of this material under different dehydration and rehydration cycles are able to stabilize a crystal volume contraction and expansion ranging from -2.170 to $+1.720 \AA^{3}$; it is the largest change in volume for a single crystal-to-single crystal transformation to date. ${ }^{[4]}$ The mechanism of this surprising all-inorganic crystal-to-crystal transformation is currently attributed to the stability of $\left\{\mathrm{P}_{8} \mathrm{~W}_{48}\right\}$ 
ring-shaped clusters and their ability to reorganize within the crystal lattice. Theory could help to understand the mechanism behind this favorable forming and breaking of $\mathrm{W}-\mathrm{O}(\mathrm{W})$ and $\mathrm{Co}-\mathrm{O}(\mathrm{W})$ bonds between the linkers and inorganic rings in the crystal lattice. In this sense, Oliveri and Overlade $^{[36]}$ applied stochastic optimizations to find metamaterials able to adapt to a sudden change in shape of a structural component under load (also known as buckling). By introducing a general optimization tool, they solved this inverse design problem and were able to explore a wide range of material properties. Their method is flexible enough, allowing us to explore different properties of metamaterials, such as band gap behavior of acoustic or photonic materials, and perhaps a similar approach could be applied to POMzites.

Given the size and the characteristics of their unique porous environment, macroscale modeling of POMzite architectures would be necessary for: (a) correlating the dimensionality of the framework and the different TM-linkers; (b) understanding bulk framework properties of the material, such as small VOCs or water absorption; and (c) modeling POMzite behaviors. POMzites are a challenging system to model and not yet fully accessible for electronic structure calculations. This task could be carried out by a dispersion-corrected density functional tight binding (DFTB) algorithm. DFTB is an approximate method based on the density functional that does not require large amounts of empirical parameters. ${ }^{\text {[3] }}$ In fact, DFT, tight binding, force fields, and continuum methods are ideal for studying large inorganic compounds, such as $>100$ transition metal atoms. The pros and cons of the DFTB are a legacy from DFT as DFTB parameters are obtained from DFT calculations. ${ }^{\text {[37] }}$ Given their structural diversity and applications, POMzites (one-dimensional (1-D), 2-D, 3-D structures) are ideal candidates to be studied with semiempirical quantum mechanical methods. Grimme et al. have developed DFTB parameters for 3-D metals to treat molecular as well as periodic systems. ${ }^{[38]}$ In fact, developing new and improved parameters for 3-D metals (eg, W, Mo, V) is a hot topic as proven by this recent publication reporting the first DFTB parameters for uranium, oxygen, and hydrogen. ${ }^{[39]}$ Developing and improving predictive computational models for transition metal chemistry in solution is an integral part of understanding their speciation under different conditions. Advances in this area will require a combination of experimental inputs with computational modeling to imagine a wide range of different rearrangements and to create a blueprint for building transition metal-based materials (TM-based).

In a recent report, Kim et al. demonstrated a successful case of inverse design of porous materials by producing 121 new crystalline porous materials using a training set of 31713 known zeolites. ${ }^{[40]}$ Their new neural network has taken the input form of energy and materials dimensions to produce reliable zeolites with a user-required range of $4 \mathrm{~kJ} \mathrm{~mol}^{-1}$ methane heat of adsorption. They used generative adversarial networks to produce crystalline porous materials given its enhanced capacity to produce realistic objects such as human faces. While this study is limited to silicon and oxygen atoms, it lays the foundation for using artificial neural networks to target user-desired properties. It is to be anticipated that the design of more complex crystalline materials, such as MOFs or POMzites, would also be feasible.

In molecular metal oxides, the current understanding of self-assembly is limited to low nuclearity clusters, and the design of new clusters is almost impossible at nuclearities greater than $\left\{\mathrm{M}_{12}\right\}$ due to a combinatorial explosion. ${ }^{[41]}$ Petrus et al. developed a computational methodology to identify the reaction mechanism for the formation of metal oxide clusters. ${ }^{[42]}$ Their work offers a speciation model based on the first principles in an automated manner and may shed light on the complexity of metal oxide self-assembly mechanisms. In this sense, and using a stochastic model, Miras et al. proved an autocatalytic network involving molecular recognition and assembly processes in the formation of molecular nanostructures of molybdenum blue. ${ }^{[43]}$ Their result solves the mystery of how gigantic clusters, such as $\left\{\mathrm{Mo}_{368}\right\}$, can form spontaneously when a simple inorganic salt of sodium molybdate is reduced under acidic conditions.

\section{4 | OUTLOOK}

The challenges presented here are about developing the tools to understand POMzites at a molecular level. The properties of framework materials are closely related to their composition and bulk state at the molecular level. POM-based frameworks are promising materials; they combine the flexibility and tuneability of redox and catalytic active porous materials with the thermal robustness owed to their tungsten framework. DFTbased simulations will be key to developing a new conceptual approach for the fabrication of functional nanomolecules and adaptive materials. Inverse design is a more advanced approach than simply using optimization algorithms and automating the search for a structure. The goal is to have a chemical property input with its desired performance metrics and allow for the algorithm to generate the best possible structure. The ultimate test for inverse design methodologies in chemistry is the synthesis, characterization, and evaluation the properties of a new material. None of what we do is in a vacuum, and the era of relying on serendipity to advance materials discovery is coming to an end. Given the current challenges already faced by chemistry, we added the 2020 global pandemic. To thrive during this uncertain period, we may need to adapt human labor to stricter shifts and increase the use of personal protective equipment. Furthermore, material scarcity will require greater planning and a goaloriented search for new materials. Inverse design in metal oxides has the potential to answer the 200-year-old question of the self-assembly mechanisms in POMs but has much more to offer. Inverse design can help in finding a specific catalyst for a zero-carbon fertilizer fuel or in obtaining a material for a new memory device able to write data more efficiently to reduce wear associated with read/write cycles and increase its lifetime. This quarantine and reflecting time have been a wake-up call, and we need to adapt our research methodologies to be more effective. Chemists will rise again to this challenge, and as a community, we will offer a new generation of materials. 


\section{ACKNOWLEDGMENTS}

Laia Vilà-Nadal acknowledges Prof Lee Cronin, Prof Mark Murrie, and Dr Edward Lee for useful discussions and proofreading of the manuscript. Laia Vilà-Nadal acknowledges EPSRC (EP/S030603/1) for funding and the University of Glasgow Early Career Development Programme (ECDP) for support.

\section{AUTHOR CONTRIBUTIONS}

Laia Vila-Nadal: Conceptualization; data curation; formal analysis; funding acquisition; investigation; methodology; project administration.

\section{ORCID}

Laia Vilà-Nadal (D) https://orcid.org/0000-0002-7718-7227

\section{REFERENCES}

[1] T. Boyd, S. G. Mitchell, D. Gabb, D.-L. Long, Y.-F. Song, L. Cronin, J. Am. Chem. Soc. 2017, 16, 5930.

[2] R. Contant, in Inorganic Syntheses (Ed: A. P. Ginsberg), John Wiley \& Sons, New York 1990, p. 110.

[3] C. Zhan, J. M. Cameron, D. Gabb, T. Boyd, R. S. Winter, L. Vilà-Nadal, S. G. Mitchell, S. Glatzel, J. Breternitz, D. H. Gregory, D. L. Long, A. MacDonell, L. Cronin, Nat. Commun. 2017, 8, 14185.

[4] L. Vilà-Nadal, L. Cronin, Nat. Rev. Mater. 2017, 2, 17054.

[5] S. G. Mitchell, C. Streb, H. N. Miras, T. Boyd, D.-L. Long, L. Cronin, Nat. Chem. 2010, 2, 308.

[6] D. L. Long, R. Tsunashima, L. Cronin, Angew. Chem. Int. Ed. 2010, 49, 1736.

[7] M. Martin-Sabi, J. Soriano-López, R. S. Winter, J. J. Chen, L. Vilà-Nadal, D. L. Long, J. R. Galán-Mascarós, L. Cronin, Nat. Catal. 2018,1 , 208.

[8] C. Busche, L. Vilà-Nadal, J. Yan, H. N. Miras, D.-L. Long, V. P. Georgiev, A. Asenov, R. H. Pedersen, N. Gadegaard, M. M. Mirza, D. J. Paul, J. M. Poblet, L. Cronin, Nature 2014, 515, 545.

[9] M. Nyman, Coord. Chem. Rev. 2017, 352, 461.

[10] C. Bo, F. Maseras, N. López, Nat. Catal. 2018, 1, 809.

[11] K. Alberi, M. B. Nardelli, A. Zakutayev, L. Mitas, S. Curtarolo, A. Jain, M. Fornari, N. Marzari, I. Takeuchi, M. L. Green, M. Kanatzidis, M. F. Toney, S. Butenko, B. Meredig, S. Lany, U. Kattner, A. Davydov, E. S. Toberer, V. Stevanovic, A. Walsh, N. G. Park, A. Aspuru-Guzik, D. P. Tabor, J. Nelson, J. Murphy, A. Setlur, J. Gregoire, H. Li, R. Xiao, A. Ludwig, L. W. Martin, A. M. Rappe, S. H. Wei, J. Perkins, J. Phys. D: Appl. Phys. $2019,52,013001$.

[12] D. Alan, Nat. Methods 2007, 4, 527.

[13] M. L. Green, C. L. Choi, J. R. Hattrick-Simpers, A. M. Joshi, I. Takeuchi, S. C. Barron, E. Campo, T. Chiang, S. Empedocles, J. M. Gregoire, A. G. Kusne, J. Martin, A. Mehta, K. Persson, Z. Trautt, J. Van Duren, A. Zakutayev, Appl. Phys. Rev. 2017, 4, 011105.

[14] J. E. Saal, S. Kirklin, M. Aykol, B. Meredig, C. Wolverton, JOM 2013, 65, 1501.

[15] V. Ozolins, E. H. Majzoub, C. Wolverton, J. Am. Chem. Soc. 2009, 131, 230.

[16] C. Ortiz, O. Eriksson, M. Klintenberg, Comput. Mater. Sci. 2009, 44, 1042.

[17] J. Greeley, T. F. Jaramillo, J. Bonde, I. Chorkendorff, J. K. Nørskov, Nat. Mater. 2006, 5, 909.

[18] N. Mounet, M. Gibertini, P. Schwaller, D. Campi, A. Merkys, A. Marrazzo, T. Sohier, I. E. Castelli, A. Cepellotti, G. Pizzi, N. Marzari, Nat. Nanotechnol. 2018, 13, 246

[19] L. Yu, A. Zunger, Phys. Rev. Lett. 2012, 108, 068701.

[20] A. D. White, G. A. Voth, J. Chem. Theory Comput. 2014, 10, 3023.

[21] W. M. Jacobs, A. Reinhardt, D. Frenkel, Proc. Natl Acad. Sci. USA 2015, 112, 6313.

[22] B. Sanchez-Lengeling, A. Aspuru-Guzik, Science 2018, 361, 360.

[23] A. Rodríguez-Fortea, N. Alegret, A. L. Balch, J. M. Poblet, Nat. Chem. 2010, 2, 955.

[24] S. C. Glotzer, M. J. Solomon, Nat. Mater. 2007, 6, 557.

[25] Q. Zheng, L. Vilà-Nadal, Z. Lang, J. J. Chen, D. L. Long, J. S. Mathieson, J. M. Poblet, L. Cronin, J. Am. Chem. Soc. 2018, 140 , 2595.

[26] L. Vilà-Nadal, S. G. Mitchell, D. L. Long, A. Rodríguez-Fortea, X. López, J. M. Poblet, L. Cronin, Dalt. Trans. 2012, 41, 2264.

[27] X. López, J. J. Carbó, C. Bo, J. M. Poblet, Chem. Soc. Rev. 2012, 41, 7537.

[28] X. López, P. Miró, J. J. Carbó, A. Rodríguez-Fortea, C. Bo, J. M. Poblet, Theor. Chem. Acc. 2011, 128, 393.

[29] J. Cameron, J. Gao, L. Vilà-Nadal, D.-L. Long, L. Cronin, Chem. Commun. 2014, 50, 2155.

[30] L. Chen, K. A. San, M. J. Turo, M. Gembicky, S. Fereidouni, M. Kalaj, A. M. Schimpf, J. Am. Chem. Soc. 2019, 141, 20261.

[31] L. Chen, M. J. Turo, M. Gembicky, R. A. Reinicke, A. M. Schimpf, Angew. Chem. Int. Ed. 2020, 59, 16609.

[32] N. Claussen, B. A. Bernevig, N. Regnault, Phys. Rev. B 2020, 101, 245117.

[33] M. Hirn, S. Mallat, N. Poilvert, Multiscale Model Simul 2017, 15, 827.

[34] J. Paik, Nature 2017, 541, 296.

[35] J. T. B. Overvelde, J. C. Weaver, C. Hoberman, K. Bertoldi, Nature 2017, 541, 347.

[36] G. Oliveri, J. T. B. Overvelde, Adv. Funct. Mater. 2020, 30, 1.

[37] A. F. Oliveira, G. Seifert, T. Heine, H. A. Duarte, J. Braz. Chem. Soc. 2009, 20, 1193.

[38] S. Grimme, C. Bannwarth, P. Shushkov, J. Chem. Theory Comput. 2017, 13, 1989.

[39] R. K. Carlson, M. J. Cawkwell, E. R. Batista, P. Yang, J. Chem. Theory Comput. 2020, 16, 3073.

[40] B. Kim, S. Lee, J. Kim, Sci. Adv. 2020, 6, 1.

[41] L. Vilà-Nadal, S. G. Mitchell, A. Rodríguez-Fortea, H. N. Miras, L. Cronin, J. M. Poblet, Phys. Chem. Chem. Phys. 2011, $13,20136$.

[42] E. Petrus, M. Segado, C. Bo, Chem. Sci. 2020, 11, 8448.

[43] H. N. Miras, C. Mathis, W. Xuan, D. L. Long, R. Pow, L. Cronin, Proc. Natl. Acad. Sci. USA 2020, 117, 10699. 


\section{AUTHOR BIOGRAPHY}

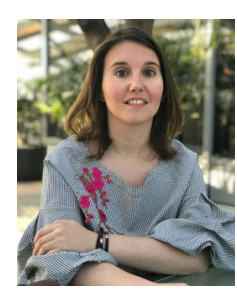

Laia Vilà-Nadal completed an MSc in Theoretical and Computational Chemistry at the Rovira i Virgili University (Spain). In 2011, she was awarded a PhD from the same university under supervision of Prof J. M. Poblet and Dr RodríguezFortea; in her thesis, she studied the step-by-step building-block mechanisms for small, 6 to 12, metal clusters, tungsten, and molybdenum oxides. In the same year, she moved to the University of Glasgow (United Kingdom) working with Prof Lee Cronin on a project that found a new memory device based on a W-Se molecular metal oxide. In 2019, she was appointed as a Lecturer (PI). In her first independent academic position, she established the Laia Vilà-Nadal group (LVNgroup) to explore the broad topic of self-assembly in metal oxides and study their properties as porous materials, memory devices, and energy storage.

How to cite this article: Vilà-Nadal L. POMzites: A roadmap for inverse design in metal oxide chemistry. Int J Quantum Chem. 2021;121:

e26493. https://doi.org/10.1002/qua.26493 